Effect of Thrombolysis on Symptom Control

On admission to the hospice, the patient contined to complain of mild to moderate dyspnoea but had no pain. He was commenced on regular immediate-release morphine $(10 \mathrm{mg}$ QDS) for breathlessness but only required one breakthrough dose of analgesia before his condition deteriorated. The patient died comfortably nine days after thrombolysis treatment.

Conclusion Thrombolysis was very effective in controlling pleuritic chest pain and acute severe dyspnoea in this patient with PE and advanced cancer with a limited life expectancy.

\section{P-86 DOES SOCIOECONOMIC STATUS AFFECT PLACE OF DEATH FOR PATIENTS KNOWN TO SPECIALIST PALLIATIVE CARE SERVICES?} ${ }^{1}$ Mike Macfarlane, ${ }^{2}$ Emma Carduff. 'West Midlands Deanery, Telford, UK; ${ }^{2}$ Marie Curie
Hospice, Glasgow, UK

\subsection{6/bmjspcare-2017-00133.85}

Background Socioeconomic (SE) status can influence where patients die, although no previous studies have investigated whether this is true following referral to specialist palliative care (SPC) services. This study investigated whether SE group influenced place of death for patients known to SPC.

Methods Place of death and SE group (using postcode data from the Scottish Index of Multiple Deprivation - SIMD) were examined in 485 patients who had died and were known to SPC services within NHS Lothian. Records were excluded if patients had died in a care home or SIMD data were unavailable. A logistic regression model was fitted with place as a three level factor and SE group as a linear regressor. The significance of differences was tested by a likelihood ratio test and contrasts assessed with Wald tests.

Results Statistically significant variation $(p=0.01)$ was found in hospital and hospice deaths between patients from the highest socioeconomic group and the lowest, although no variation was found in home deaths. Only 45\% (22/49) of patients known to SPC services in the lowest SE group died in a hospice, compared to $60 \%(97 / 163)$ in the highest, while $28.5 \%$ (14/49) of patients known to SPC from the lowest SE group died in hospital compared to $12 \%(20 / 163)$ in the highest.

Conclusions Even after referral to SPC, statistically significant variation in place of death was found between patients from the highest socioeconomic group and the lowest. Patients from the highest SE group were more likely to die in a hospice and less likely to die in a hospital than patients from the lowest SE group, although no variation in home deaths was found. Further multivariate analysis and investigation of reasons for this variation are required

\section{P-87 PHYSIOTHERAPY PALLIATIVE CANCER CARE: A CASE STUDY APPROACH}

${ }^{1,2}$ Caroline Belchamber, ${ }^{2}$ Elizabeth Rosser, ${ }^{2}$ Caroline Ellis-Hill. ${ }^{1}$ Sue Ryder, London, Great Britain; ${ }^{2}$ Bournemouth University, Bournemouth, Great Britain

\subsection{6/bmjspcare-2017-00133.86}

Background Evidence suggests that there is an increasing need for physiotherapists in palliative cancer care services. However, there is a lack of understanding of their role, educational needs and place within the rehabilitation team. The purpose of this study was to explore a local palliative cancer care physiotherapy service provision in light of best practice recommendations.

Method A qualitative approach using a single case study design was chosen as it provided a framework to facilitate the inclusion of multiple perspectives in a complex context within an area of healthcare where little research has been undertaken. Multiple methods of data collection were used from numerous perspectives, which included four data sets: interviews (healthcare professionals [10]); interviews (service users [10]); observations (physiotherapists [2] treating service users [5]); and policy document collection (NICE guidelines; white papers [12]). Data collection occurred from 2008 to 2014 and detailed data analysis was carried out from 2012 to 2014 using a thematic approach within a framework, comparing and contrasting patterns within and across the four data sets.

Findings Emergent themes highlighted a number of important aspects relating to physiotherapists including: new ways of working, shift in mindset and treatment planning, emotional adjustment and integration of professional boundaries. This evidenced a metamorphosis of both the physiotherapist's profession and service provision through key competencies, such as entrepreneurial, leadership, policy champion and humanisation skills, which enabled them to meet essential policy recommendations of service quality and innovation.

Conclusion This study provides a framework and unique perspective within which the physiotherapy profession can focus its attention on how physiotherapy needs to change in the future. However, a debate is necessary around the need for profession specific or service specific outcomes in this area, and how physiotherapists 'prove their worth' now that they are an integral part of palliative cancer care provision.

\section{P-88 EVALUATION OF A PILOT SERVICE DESIGNED TO IMPROVE TRANSITION FROM CHILDREN'S PALLIATIVE CARE SERVICES}

Sinead Hutcheson, Clare White, Hilary Maguire. Northern Ireland Hospice, Belfast, UK

\subsection{6/bmjspcare-2017-00133.87}

Background There are increasing numbers of young people (YP) with life limiting diagnoses living into adulthood. When these YP become too old for children's hospice services there is a recognised gap in their care and they often experience challenges when transitioning into adult services. A two year pilot project was developed between a hospice's adult and children's services to try to address this gap and aid transitioning. The pilot involved a day service two Saturdays per month with occasional overnight trips, with two separate groups depending on the YP's ability to communicate.

Aim To evaluate the pilot project.

Method Three focus groups made up of key stakeholders in the service (YP, their parents and staff) were performed and analysed using an adopted thematic analysis framework.

Results Three YP, seven parents and six staff members participated. Five main themes across the three groups were identified;

- Transition is Tough

- Continuity

- Parental Care

- The pilot service helps

- The Future 